\title{
LECTURA, ENSEÑANZA DE LENGUA Y DESERCIÓN ESTUDIANTIL EN SORDOS Y UNIVERSITARIOS
}

\author{
Juan Andrés Larrinaga*
}

\begin{abstract}
RESUMEN
En este trabajo se analiza la relación de los estudiantes sordos y de los estudiantes universitarios ingresantes en lo concerniente a la lectura de textos académicos, se muestran los similares procesos de exclusión que ocurren sobre ambos grupos, se vinculan dichos procesos con la educación lingüística, básicamente en torno a las prácticas de lectura, y finalmente se reflexiona sobre las intervenciones del sistema educativo en la formación lingüística. Estos análisis y consideraciones teórico-metodológicas que proponemos surgen a punto de partida de algunos resultados de la investigación que venimos llevando adelante en diversas Facultades del Área Social de la UdelaR, a través de la instrumentación de cursos de comprensión lectora en inglés, y del trabajo sobre la educación de los sordos en primaria y secundaria.
\end{abstract}

Palabras clave: Lectura. Enseñanza de lengua. Deserción estudiantil.

\begin{abstract}
In this paper I analyze the relationship between Deaf students and some university students of the entering generation with regard to their reading of academic texts and show the process of exclusion both groups undergo from the education system. I relate that exclusion to their linguistic education, more specifically to their reading practices. Finally, I introduce some reflexions on possible interventions of the system of education in their linguistic education. The following considerations have come up in the course of a research I have been carrying out in various Schools from the Social Area of the UdelaR mainly in reading courses of academic texts in English, and in the education of the Deaf in primary and secondary school.
\end{abstract}

Key words: Reading. Language teaching. Student dropout.

\footnotetext{
* Doctorando en Educación en la Universidad Nacional de Entre Rios (UNER), Argentina. Profesor Adjunto del Área Lengua de la Facultad de la Información y de la Comunicación y Profesor Adjunto de Inglés en Traductorado de la Facultad de Derecho de la Universidad de la República (UdelaR).E-mail: jalarrin@gmail.com
} 


\section{Introducción}

El procesamiento de la lengua escrita, y en especial la lectura, es de primordial importancia en el sistema educativo formal. El objetivo de este trabajo es presentar algunos temas de relevancia relativos a la educación lingüística de dos grupos insertos en el sistema público uruguayo de enseñanza: los estudiantes sordos, entendidos como los hablantes de la LSU (lengua de señas uruguaya) y los estudiantes oyentes ingresantes a la Universidad.

Estos dos grupos tienen un problema en común: les es difícil mantenerse y progresar dentro del sistema formal de educación. Entre las razones de este problema podemos señalar las dificultades, en mayor o menor medida dependiendo de los casos individuales, en el procesamiento de los materiales de lectura que deben comprender para progresar en el sistema.

Los estudiantes universitarios representan una población que ha crecido enormemente en el último siglo. Lo que sorprende en nuestra universidad, la Universidad de la República (UdelaR) son los altos índices de deserción estudiantil: en muchas Facultades sólo entre el 10 y el 20\% de los estudiantes que ingresan se gradúa. La mayoría de ese alto porcentaje de estudiantes que abandona sus estudios lo hace luego de experiencias frustrantes en la etapa inicial de sus estudios (DICONCA, 2011).

Por su parte, los estudiantes sordos han pasado con dificultades la etapa primaria de educación formal, pero han sido históricamente excluidos de los otros niveles educativos (ANEP, 2008, 2009, 2010, 2011, 2012, 2013; PELUSO, 2010). Tradicionalmente la lengua natural de los sordos, las diferentes lenguas de señas que discurren por la materialidad viso-espacial, no eran reconocidas como lenguas y las prácticas oralistas ocupaban un alto porcentaje de los esfuerzos educativos. Esto mantenía al grupo alejado de la producción de conocimiento y de las prácticas letradas relativas a la actividad cultural, académica y científica.

Solo recientemente las lenguas de señas han sido reconocidas como tales y la educación bilingüe ha pasado a tener un lugar en la educación formal (aunque a veces con un rol menor), por lo que esta situación, de a poco, se viene revirtiendo (BEHARES, 1989; PELUSO, 2010; LARRINAGA; PELUSO, 2007; BEHARES; BROVETTO; PELUSO, 2012). Uno de los problemas que enfrentan las lenguas de señas para ser vistas como tales en la educación es el hecho de que no tienen forma escrita como las lenguas 
orales. Con tal historia, el acceso a la educación formal de la comunidad sorda ha estado muy limitado y aún hoy en pocos lugares pueden verse miembros de esta comunidad en los niveles más altos de la educación formal (ANEP, 2013).

Nos interesa particularmente relacionar la mencionada exclusión de estos dos grupos con la educación lingüística, enfocándonos en las prácticas de lectura, al tiempo que reflexionaremos sobre posibles formas de mejorar o incrementar las intervenciones del sistema educativo en la formación lingüística.

Cabe señalar, que en este trabajo se recogen algunos resultados de la investigación que venimos llevando adelante en diversas Facultades del Área Social de la UdelaR. Se trabajó con la población universitaria en varias Facultades a punto de partida de los cursos de comprensión lectora en inglés y con población sorda de primaria y secundaria. También fueron estudiadas las representaciones que docentes de estos diversos contextos educativos tenían hacia las habilidades lectoras de los estudiantes. Esta investigación fue un antecedente importante para la actual investigación que co-coordino, en la que se busca indagar las actitudes y soluciones de los docentes frente a los problemas que presentan la lectura y la escritura en dos servicios de la UdelaR, y que cuenta actualmente con financiación de dicha Universidad (CSIC/CSE).

\section{Los estudiantes universitarios, los estudiantes sordos y el español escrito}

Como ya señalamos, las dos poblaciones que se tratarán en este trabajo son los estudiantes sordos y algunos estudiantes oyentes ingresantes a la Universidad, dado que presentan problemáticas en relación a la lectura académica y eso determina contextos de exclusión del sistema educativo.

La Universidad de la República era la única Universidad en el país hasta fines de los años ochenta cuando empezaron a aparecer instituciones privadas. Como Universidad nacional y pública, tiene entre sus metas la creación de conocimiento, la formación de profesionales y la extensión. La UdelaR es la Universidad más importante del país, no solo por su carácter gratuito sino también por las actividades de extensión que consisten en la realización de actividades en el medio, incluyendo los servicios que ofrece en los campos de la salud y la asistencia legal que cubren la mayoría de 
las necesidades de gran parte de la población de bajos ingresos. Además, realiza acuerdos con variadas entidades públicas y privadas a las que ofrece asesoramiento técnico. Pero lo más relevante es que la UdelaR concentra entre el 70 y $80 \%$ de la actividad científica del país y posee alrededor de 100.000 estudiantes en un país que cuenta con una población de algo más de tres millones (UDELAR, 2013).

Sin embargo, como fue mencionado anteriormente, existe un alto porcentaje de deserción estudiantil. La UdelaR, que permite a todos los ciudadanos del país continuar sus estudios luego de finalizada la etapa secundaria, termina por excluir una parte importante de esa población (DICONCA, 2011; CSE/UDELAR, 2014). Asimismo, variadas pruebas diagnósticas aplicadas en diversos sectores de nuestro sistema educativo señalan que la educación en el área lengua es un sector débil que requiere más trabajo, lo que podría mejorar la situación (MACRI; NOBOA, 2004; BARCO; LIZARRITURRI, 2005; TORRES; FEDORCZUK; VIERA, 2009).

La constatación de esta situación llevó a que la Comisión Sectorial de Enseñanza de la UdelaR implementara el curso "Comprensión y Producción de textos en la Universidad" destinado a docentes de todos los Servicios de la UdelaR y que tenía, por objetivo, la reflexión crítica en relación a la lectura y escritura en la Universidad, así como la promoción de la incorporación de estrategias de trabajo para ayudar a los estudiantes en su manejo con los textos escritos y la replicación de este conocimiento hacia otros docentes (BARCO; LIZARRITURRI, 2005).

Similares dificultades a las que se señalan en el manejo de textualidad académica por parte de los estudiantes universitarios de UdelaR se observan en otras Universidades de la Región, como ser las de Chile, Argentina y Colombia (ARNOUX, 2004; CARLINO, 2005; CISNEROS, 2007; DESINANO, 2009; PARODI, 2010; SCHWEIZER, 2011; CARTOLARI; CARLINO, 2012).

En consonancia con todos estos estudios referidos, tanto para Uruguay como para la Región, en las Facultades del Área Social en que se ha desarrollado mi investigación educativa a través de los cursos de comprensión lectora en inglés también se constató que las dificultades que presentan los estudiantes a la hora de enfrentarse a los textos académicos en dicha lengua no se refieren exclusivamente al manejo de la lengua extranjera, sino principalmente, al manejo de este tipo de textualidad (LARRINAGA, 2003). 
Cuando se trabaja en inglés en los niveles de cohesión y coherencia de los textos ofrecidos, académicos y periodísticos, se observa que los estudiantes muchas veces no reconocen estos aspectos en su propia lengua en relación a estos particulares textos, que son los que necesitan poder leer para mantenerse dentro de sus estudios terciarios. También se puede observar cómo los estudiantes manejan aspectos más básicos que la cohesión y la coherencia, tales como la morfología y la sintaxis. Aquellos estudiantes con manejo de la morfología y la sintaxis más creativo y asociativo se permiten más "flexibilidades" interpretativas a la hora de la lectura.

Estas dificultades son claramente una de las razones probables de la frustración y la deserción de parte del estudiantado. Si bien es obvio que no se puede relacionar de forma directa y exclusiva los problemas que presentan los estudiantes con la lectura y escritura de textos académicos con su deserción, creemos que este es un factor que seguramente puede hacer su trayectoria por el sistema más fácil o más difícil.

Por su parte, cuando hablamos de la educación para la comunidad sorda en nuestro país de deben tener presentes cuatro conceptos: lectura, equidad, empoderamiento y accesibilidad (LARRINAGA; PELUSO, 2009; URUGUAY, 2008,2009; PELUSO, 2011). Al discutir estos conceptos debemos recordar que la mayoría de las lenguas de señas (lenguas naturales de las comunidades sordas) no han desarrollado formas escritas a la manera que lo han hecho las lenguas orales, lo que ha tenido claras consecuencias en los procesos educativos de los sordos.

Al trabajar con grupos minoritarios, como el de los sordos, debemos manejar los parámetros que aseguren para ellos equidad en el sistema de educación pública. Esto significa acceso temprano y fluido a la lengua de señas nacional o de la comunidad, empoderamiento a través de la creación de productos culturales en esa lengua de señas, la búsqueda de sistemas alternativos de registro de las lenguas de señas (PELUSO, 2011), y la enseñanza de habilidades lectoras (en principio en español, en el caso de los sordos uruguayos).

En el mundo actual, desde cualquier lengua y cultura, la lectura es necesaria para asegurar la accesibilidad a la información, y para la mayoría de los campos tecnológicos y científicos se necesita, inclusive, leer en más de una de las lenguas en que dicho conocimiento aparece, lenguas de poder a veces llamadas variedades superestándar (TRUDGILL; HANNAH, 2013). 
Es por esta razón que leer en español es altamente relevante para los sordos en Uruguay: no solo porque es una de las lenguas nacionales, sino porque también es la lengua en la que los últimos desarrollos del conocimiento son registrados en nuestro país y en gran parte del mundo.

En el marco de la educación bilingüe de los sordos, las relaciones entre oralidad y escritura son un tema de particular interés. En una situación de bilingüismo clásico, hay ocho habilidades psicolingüísticas en juego que en nuestro caso, en Uruguay, serían: comprensión del español oral; producción del español oral; comprensión del español escrito; producción del español escrito; comprensión de la LSU oral, producción de la LSU oral; comprensión de la LSU escrita y producción de la LSU escrita.

Pero esto presenta ciertas particularidades en el caso de los sordos: básicamente no se desarrolla el español en la oralidad ni la LSU en la escritura. Por un lado debido a que la oralidad en español está fuertemente interferida en los sordos porque no tienen acceso a la lengua oral a través del canal oral. Por otro lado debido a que la LSU no tiene hasta ahora escritura. De esta forma en Uruguay la educación bilingüe de los sordos trata de adaptarse a este particular bilingüismo. Es decir toma en cuenta que las lenguas involucradas - español y LSU - mantienen una distribución funcional de acuerdo a la cual la LSU se especializa en la oralidad (interacciones cara a cara) y el español en la escritura. Esto ocurre básicamente en Montevideo y en algunas capitales departamentales. En estos contextos educativos las clases se imparten en LSU (directamente por los docentes que son bilingües o a través de un intérprete) pero la bibliografía a ser estudiada está escrita en español.

Debido a lo anteriormente señalado, las prácticas de cada docente en su área incluyen una fuerte reflexión lingüística, ya que cada docente tiene que ayudar a sus estudiantes a leer los materiales escritos sobre el tema que ellos enseñan, en una segunda lengua (el español) y entonces los requerimientos metalingüísticos se duplican.

Esta particular especialización del bilingüismo desafía los modelos de enseñanza de la lectura y escritura en español en este caso ya que deben ser enseñadas sin el apoyo de la oralidad en esta lengua. 


\section{Los docentes de las asignaturas como docentes de lengua}

En una clase tradicional para estudiantes oyentes una de las herramientas de enseñanza que los docentes han estado usando por siglos para enseñar lectura y escritura es el discurso pedagógico (LARRINAGA, 2003; LARRINAGA; PELUSO, 2004).

En este sentido, si pensamos por ejemplo en el nivel de educación secundaria, es la etapa en la que los estudiantes comienzan propiamente a estudiar de la forma en la que lo deberán hacer de ahí en más en todo el sistema educativo formal. Generalmente en la escuela primaria los estudiantes hacen tareas o deberes, pero el estudio del texto disciplinar no es aún tan relevante. Para ayudar a los estudiantes a enfrentar las dificultades en la lectura de sus primeros textos científicos, encontraremos a los docentes usando su más valorada herramienta: su discurso oral.

Desde el punto de vista del discurso pedagógico, podemos interpretar esta situación como que la tarea del docente en esta etapa es asegurar la entrada del estudiante al discurso científico de la materia en cuestión. Aunque el discurso del docente en la clase es altamente variable, con momentos de mayor informalidad, también contiene momentos en que, por razones pedagógicas, el docente introduce un discurso muy cercano al formato de lengua escrita.

Este recurso educativo que consiste en moverse discursivamente dentro de la oralidad hacia el polo formal, típico de la escritura, ayuda a los estudiantes, tanto funcional como estructuralmente, a leer los textos escritos académicos de la disciplina. Los buenos docentes no solo trasmiten los contenidos que quieren enseñar sino que también son conscientes del hecho de que sus estudiantes tendrán luego que manejar textos complejos para continuar estudiando la disciplina. Los docentes intuitivos en algunos momentos especiales de la clase se encontrarán estructurando su discurso pedagógico a la manera de la lengua escrita, para ayudar a sus estudiantes a franquear la distancia entre la oralidad y la escritura.

Esto es lo que sucede con los estudiantes oyentes. Los estudiantes sordos no tienen acceso directo a la estrategia del docente para andamiar (BRUNER, 1984) la lectura, modelando su discurso a la manera de un texto escrito, por lo tanto para los sordos el procesamiento de los textos en español sin una oralidad en esta lengua se torna doblemente más difí- 
cil que para un estudiante oyente. En primer lugar porque no dominan la lengua en la que está el texto, en tanto no es su primera lengua, como sí lo hacen los estudiantes oyentes. En segundo lugar porque no tienen acceso a la estrategia desplegada por el docente para andamiar la lectura a través de la sofisticada estructuración de su discurso. Las explicaciones en LSU nunca resultarán tan transparentes dado que son en otra lengua diferente a aquella en que están escritos los textos y que sólo cuenta con el canal oral, pues no tiene escritura.

\section{Relevancia y complejidades de la lectura}

Algunos desarrollos tecnológicos relativamente recientes en nuestra cultura occidental han hecho competir a la lengua escrita con medios audiovisuales. Si pensamos en los individuos, algunas generaciones atrás, vemos que tenían tiempos de calmada lectura más largos que en la actualidad, dado que ciertas funciones se cumplían casi exclusivamente a través de la lectura: como por ejemplo la información (diarios, semanarios, revistas, etc.). Lo mismo sucedía con el entretenimiento (cuentos, novelas, etc.) y con el estudio.

Las computadoras y los medios audiovisuales resultan ser vías culturales válidas para tales actividades y que agregan variedad, pero al mismo tiempo esta tendencia deja a las nuevas generaciones con menos chances de practicar y desarrollar la lectura. Esto no sería un problema si pudiéramos dejar de lado la lengua escrita pero, como sabemos, esto no es así por muchas razones.

Otros desarrollos recientes en nuestra cultura han hecho que la lengua escrita adquiera nueva relevancia. Hace un siglo, o algo más, la educación formal en cualquiera de sus niveles era para una elite y la integración masiva al nivel terciario de la educación es un fenómeno relativamente reciente. En nuestra actual cultura altamente sofisticada, compleja y competitiva, necesitamos ser capaces de mantenernos dentro del sistema formal de educación $\mathrm{y}$, si es posible, asistir a la Universidad.

El manejo de las prácticas letradas ya no consiste solo en un tema de enseñanza primaria o secundaria $\mathrm{o}$, en la vida adulta, en la posibilidad de leer instrucciones o un artículo en el diario. Ser letrado también involucra, ahora, para muchos, manejar artículos técnicos altamente sofisticados en 
nuestra lengua nativa y en al menos otra lengua internacional. Para procesar información en campos de alta complejidad, para leer y estudiar textos estructural y semánticamente densos, las prácticas lectoras en más de una lengua, con todas las estrategias que involucran, continúan siendo relevantes. En los ámbitos académicos y técnicos la lectura es de enorme importancia.

En cuanto a la complejidad de la lectura, cabe señalar que se lee a diferentes ritmos dependiendo de las características y posibilidades personales y también según la situación, los tipos de texto y los diferentes objetivos de cada lector para con el texto. En términos generales, podemos hablar de lectura analítica versus lectura general o global. Esta clasificación de las modalidades lectoras se inspira en los conceptos que ha desarrollado la psicología cognitiva de procesamiento top-down a diferencia de procesamiento bottom-up. Tal como señalan Dawson y Medler (2009, p. 37):

Information can flow both from the bottom of the system to the top of the system and from the top of the system to the bottom of the system. When information flows from the top of the system to the bottom of the system this is called "top-down processing", which is sometimes known as "theory-driven processing".

The implications of this top to bottom flow of information is that information coming into the system (perceptually) can be influenced by what the individual already knows about the information that is coming into the system (as information about past experiences are stored in the higher levels of the system).

Por lo tanto, de acuerdo a la psicología cognitiva, los procesos top-down favorecen un tipo de comprensión más influenciada por el conocimiento y las experiencias previas. La lectura global es una modalidad lectora que descansa fuertemente sobre el conocimiento previo y que mecánicamente depende más del rápido reconocimiento léxico que de la decodificación de relaciones intratextuales. Entonces, esta modalidad lectora, no permite una profunda penetración en el material nuevo que el texto pueda contener. Al usar esta modalidad, un lector será menos afectado por lo que está leyendo ya que sus ideas previas seguirán influyendo fuertemente en su comprensión del texto. Sin embargo esta modalidad presenta una clara ventaja comparada con la modalidad analítica: aumenta sustancialmente la velocidad de lectura. 
Por otro lado, la lectura analítica es una modalidad lectora que permite una comprensión exhaustiva de un texto estructural y conceptualmente denso. Los procesos que se siguen pasan, necesariamente, por áreas de decodificación formal, sintáctica, lógica y morfológica.

Ninguna de estas modalidades es exclusiva ni excluyente, y es mejor pensarlas en términos de polaridades: en un extremo se ubicaría la lectura muy analítica con total decodificación de todas las relaciones sintácticas y una interpretación de estas; y en el otro extremo, una lectura muy global, consistente en escanear el texto en búsqueda de palabras claves o conocidas. Entre medio de estos extremos, podemos encontrar diversos estadios de decodificación borrosa. Un lector ideal se podría mover entre ambas polaridades, de acuerdo a sus necesidades, y con pleno control.

Sin embargo, en un individuo específico, una de las modalidades puede aparecer como la más frecuente o la principal. La preponderancia de una modalidad depende de muchos factores, tales como el objetivo del lector para con el texto, el tipo de texto a ser leído, los estilos lectores desarrollados por la comunidad a la que el lector pertenece (los estudiantes de medicina podrían leer más globalmente que los de ingeniería o viceversa), los condicionamientos metodológicos durante los estudios anteriores del lector, para mencionar solo algunos de los más relevantes.

Muchas veces, a pesar de factores condicionantes textuales y extra textuales una de las modalidades lectoras prevalece, y esto puede obstaculizar al lector para alcanzar las metas que se había trazado al inicio.

La lectura académica y las instancias relevantes de estudio o trabajo exigen al lector una lectura analítica, ya que éste se propone recibir grandes cantidades de información, mayormente nueva, y comprender las relaciones sutiles propuestas por el texto entre los diferentes argumentos, etc. Si el lector no consigue decodificar, por ejemplo, un conector adversativo, podría terminar comprendiendo lo opuesto de la idea que el texto pretende transmitir.

Los estudiantes al ingresar a la Universidad todavía están en proceso de desarrollo de este tipo de lectura analítica. Si algunos estudiantes no han alcanzado el nivel de competencia de la lectura analítica que se requiere para manejar la cantidad y profundidad de los materiales de lectura que se les da en la Universidad, pueden empezar a experimentar dificultades en su actuación académica. Esto es aún más dramático para el caso de los estudiantes sordos, para quienes el español escrito implica lectura en una 
segunda lengua, por lo que, como ya explicamos, es doblemente ajeno (la ajenidad de la lengua y la de los códigos escritos académicos).

Debido a la naturaleza del proceso de la lectura que tiene lugar mayormente en aislamiento y silencio, donde el lector está solo con el texto, éste puede tomarse tiempo para reflexionar y permanecer en el texto en el que siempre hay cosas complejas a descubrir. La lectura no es una función del lenguaje como la conversación, en la que hay un interlocutor con el que se negocian los significados y nos fuerza a seguir adelante. Cuando leemos vamos para atrás y para adelante todo el tiempo, principalmente cuando estamos leyendo textos técnicos en cualquier campo, dado que la información es nueva y una lectura global no sería suficiente. En ese movimiento de ida y vuelta tenemos tiempo de usar estrategias metalingüísticas, es decir usar lo que sabemos sobre la lengua. Cuanto más conscientes o intuitivamente conscientes somos de los aspectos formales del lenguaje, mejor podremos usar estas estrategias al leer y tendremos más control sobre el proceso. Si un buen lector no comprende una oración importante en la lectura, podrá retroceder, por ejemplo, en busca del referente de un pronombre que habilite la comprensión o en busca de una idea contrastante que explique la presencia de una partícula adversativa.

\section{La lectura y el acceso al conocimiento}

Como ya dijimos, los estudiantes sordos (que han sido históricamente excluidos de los niveles secundario y terciario del sistema) y los estudiantes que ingresan en la Universidad representan dos grupos con dificultades en mantenerse y progresar en el sistema educativo formal y que pueden presentar problemas en el procesamiento de los textos que deben leer para tener acceso al conocimiento. El sistema educativo ha intervenido, tradicionalmente, de forma indirecta, tratando de mejorar los procesos de lectura, pero se requiere conciencia del problema para realizar intervenciones que realmente puedan modificar esta situación.

La lectura, a diferencia de la escritura, siempre ha sido un proceso difícil de compartir, ya que es mayormente silencioso. El producto del proceso de la escritura es un texto, algo tangible y compartible. El producto de la lectura es la comprensión, algo intangible y a veces silenciado o velado por problemas pragmáticos de producción o explicación. En los procesos de enseñanza y 
aprendizaje con estudiantes sordos y estudiantes universitarios en las clases de comprensión lectora de inglés, hemos transitado diversas vías metodológicas para superar este problema de accesibilidad al proceso lector. Se pudo observar que una forma simple de ayudar a alguien a leer mejor es leer con él. Esto implica metodológicamente no dejar la lectura como un proceso solitario, silencioso, sino avanzar en el texto con el otro individuo. Esto se hace metodológicamente más complejo en el caso de los sordos y merecería un capítulo aparte, ya que, mientras que los oyentes pueden leer y escuchar el proceso del otro a través de la lectura en voz alta con comentarios sobre el proceso, los sordos deben leer y a través del mismo canal viso espacial seguir el proceso del otro.

De esta manera estamos creando una instancia que los docentes frecuentemente llamamos el "show how", emulando la expresión "knowhow" acuñada en el mundo de los negocios. Debido a que existen muchas diferentes estrategias, a veces individuales, de extraer significados del texto, la expresión "show how" refiere al hecho de que estamos mostrando las estrategias que hemos usado para comprender algo como manera de compartirlas con el otro (estudiantes universitarios o sordos). A veces leer en voz alta señalando elementos del texto, con comentarios y explicaciones, puede "enseñar" o mostrar estrategias de interpretación. La cercanía física con el otro puede ayudar pues el apuntar con el dedo ciertas palabras o marcas resulta a veces relevante.

Esta lectura para realizar el "show how" debe ser una lectura con comentarios sobre las maneras en que estamos manejado la forma de cada oración, señalando las relaciones que establecemos en todos los niveles lingüísticos. Hacer explícito el proceso de la lectura no es algo que hagamos usualmente, ya que quien lo está haciendo - tutor, docente - está "prestando" sus categorías lingüísticas y debe desplegar un alto nivel de conciencia lingüística.

Por otro lado, la lectura con compañeros es muy útil para el desarrollo del proceso lector (PROGRESA; UDELAR, 2012). Aunque un estudiante no está entrenado para explicitar su conciencia lingüística, naturalmente muestra por qué respondió una pregunta de determinada manera o cómo solucionó un problema del párrafo leído. En cierta medida, hace algún tiempo y aún hoy en algunas Facultades de nuestra Universidad, los grupos de pares que estudian juntos (un hábito tradicionalmente arraigado en nuestra Universidad) proveen la lectura en conjunto y el compartir estrategias necesarias para alcanzar mejores niveles de lectura analítica. 


\section{Ideologías lingüísticas y educativas para repensar la enseñanza de la lectura}

En las próximas páginas discutiremos tres posiciones teóricas y cómo ellas influyen en nuestras intervenciones educativas en el área lengua: la construcción de las disciplinas dentro del sistema educativo, la ideología lingüística y el plurilingüismo, y el concepto de lengua que subyace en la enseñanza de la lectura.

\section{El problema de la construcción de las disciplinas y el objeto lengua.}

Dentro de nuestro sistema educativo es interesante observar cómo las diferentes asignaturas se han conformado tradicionalmente, es decir, cómo el conocimiento es segmentado (TERIGE; DIKER, 1997; CALDERÓN, 2000). La delimitación de las disciplinas en todo el sistema educativo en el área lengua nos ha dejado con asignaturas tradicionales fijas tales como: Español, Lengua, Literatura, Inglés, Portugués, Morfosintaxis, etc. Aunque parece un panorama muy variado, en todas estas materias se presentan ciertos rasgos que responden a una rigidización del campo. Existe una clara separación y jerarquización entre lengua materna y lengua extranjera y una dicotomización entre forma y contenido, expresadas en metodologías de enseñanza a veces muy diferentes.

En general, dentro de las asignaturas así conformadas la idea de lengua como una entidad claramente circunscrita y homogénea prevalece, en parte como consecuencia de la influencia de la modernidad (una lengua una nación), de procesos identitarios (separarse de los otros) y de los procesos de estandarización de las lenguas que crean la variedad estándar que tiene una representación muy peculiar en los hablantes, creando la ilusión de que existe "La Lengua", como entidad objetiva y homogénea.

Esta idea de homogeneidad lingüística ha sido clásicamente discutida por la sociolingüística.

Los principales marcos teóricos en lingüística (SAUSSURE, 1916, 1993; CHOMSKY, 1965, 1989) han conceptualizado al objeto de la lingüística (langue en el caso de Saussure e internallanguage - language, generativegrammar, competence, knowledge of language - en el caso de Chomsky) desde una perspectiva abstracta, formal e idealizada, sosteniendo su homogeneidad. Estas teorías lingüísticas se han enfocado en explicar el 
mecanismo del lenguaje en sí y su esencia y, en definitiva, lo que significa ser individuos con lenguaje.

Con los trabajos pioneros de Labov (1966) se relaciona al lenguaje con el área social, mostrando la gran variación inherente al mismo. La sociolingüística abandona la idea de homogeneidad lingüística, muestra la gran heterogeneidad del fenómeno y brinda un marco explicativo a las relaciones entre el lenguaje y la sociedad.

Desde esta perspectiva, la lengua está formada por un sinnúmero de variedades que responden a las categorías sociales que impactan sobre ella, como, por ejemplo, edad, clase social, ubicación geográfica, sexo/género y diferentes escenarios de uso, como contextos más o menos formales, la escritura y la oralidad, etc. Las relaciones de poder que ocurren en la sociedad no solo se reproducen sino que también actúan en el lenguaje. No solo hablamos una lengua, sino que también realizamos actos de identidad en ella (LE PAGE, 1968).

Frente a esta visión del lenguaje como una entidad altamente heterogénea, la visión de sus límites también se afecta: ¿dónde comienza una lengua y termina la otra? Esta pregunta se vuelve más dramática en el caso de lenguas claramente relacionadas. Por ejemplo, ¿el español y el portugués son dos lenguas diferentes?

Las mismas preguntas se pueden hacer en sentido inverso, no desde la diferencia sino desde la unidad: ¿hasta que punto el español rioplatense y el español rural mexicano son la misma "lengua"?

Hudson (1987) se posiciona en un extremo del debate "language" vs. "variety", e introduce el concepto de "linguisticitems" que ayuda a romper la idea de límites precisos entre las lenguas. Un ítem lingüístico puede ser cualquier pieza de conocimiento lingüístico: léxica, morfológica, sintáctica, semántica o pragmática. Estos ítems lingüísticos pueden ser organizados, con fines metodológicos de estudio (o de enseñanza). Se forman así conjuntos de ítems lingüísticos a los que Hudson (1987) denominó "attenuatedforms of varieties", aunque cada hablante posee su propio conjunto, más allá de la procedencia de cada ítem. En definitiva este autor abandona el concepto de lengua por el de variedad, que sería un conjunto de ítems lingüísticos con similar distribución sociolingüística. A su vez estos conjuntos estarían en un continuo de inteligibilidad, tanto diatópico como diacrónico. El concepto de lengua permanecería como una entidad cultural antropológica. 
En este marco, la delimitación de las asignaturas del área lengua es muy compleja. A la dicotomización entre materno y extranjero, entre forma y contenido, entre ámbitos de uso formal e informal, o escrito y oral, se agrega que el propio objeto de las asignaturas es esencialmente heterogéneo y que se constituye en homogéneo para su estudio y enseñanza. Además dicha homogeneización tiene importantes componentes ideológicos y políticos: aquellos relacionados con la variedad estándar y la ideología del purismo idiomático y la corrección.

Así, las diferentes asignaturas del área lengua tienden a estar aisladas dentro de nuestro sistema educativo y no recogen estas discusiones relativas a la heterogeneidad de la lengua. Por ejemplo, cómo es posible observar en los documentos oficiales de la Comisión de Política Lingüística de la Administración Nacional de Educación Pública (ANEP, 2008), el español se enseña por un lado en asignaturas como Literatura en las que se enfatiza el contenido, pero en las que sólo lo literario constituye el contenido y, por otro lado, en asignaturas como Idioma Español en las que se enseña gramática, es decir, en las que se enfatiza la forma, desde una perspectiva fuertemente estructuralista. A su vez estas asignaturas en general no se conectan con otras, como Historia o Geografía que se concentran en el contenido y se da menor relevancia a la tarea de introducir al estudiante en esos nuevos mundos científicos, en sus campos léxicos, en las formaciones discursivas propias y en la lectura de la disciplina.

Además, la asignatura Inglés, que es la única asignatura curricular de una lengua extranjera en nuestro sistema formal de educación, es enseñada de forma relativamente desconectada de los otros esfuerzos lingüísticos que los estudiantes están realizando en las demás asignaturas a un cierto nivel.

Por su parte la asignatura Lengua de Señas Uruguaya, que está tomando existencia de forma paulatina en nuestro sistema educativo en sus tres ramas, presenta no sólo los mismos problemas ya relatados para las otras asignaturas, sino también el de ser una lengua en proceso de estandarización, que carece de escritura y de otros instrumentos de gramatización (gramáticas y diccionarios).

Así, mientras la enseñanza de los contenidos impide muchas veces el señalar fenómenos formales interesantes tanto léxicos como sintácticos o de formaciones metafóricas y otros, que se encuentran no sólo en la gran literatura o la gran ciencia sino a todos los niveles, la concentración en as- 
pectos formales con escasos contextos de uso y contextos contrastivos lleva a la presentación del "objeto lengua" en forma poco significativa.

\section{Políticas lingüísticas e ideologías lingüísticas}

Las decisiones relativas a la educación lingüística en el contexto de la educación formal y cómo se establecen las relaciones entre las lenguas y las habilidades específicas a enseñar, claramente responden a las ideologías lingüísticas que han prevalecido en los diferentes períodos socio-históricos, las que en general tienen un carácter global.

Las ideologías lingüísticas pueden ser agrupadas en torno a dos grandes ejes: las ideologías monoglósicas y las ideologías pluriglósicas (DEL VALLE, 1999). Por ideología monoglósica se entiende aquella que sostuvo todo el proyecto modernista europeo, que imponía de forma fuerte la noción: una nación - una lengua. Es una ideología que se basa en la idea de la homogeneidad y de la construcción de la identidad nacional bajo ese concepto. Según esta ideología modernista, todos los habitantes de un país hablan la misma lengua de forma homogénea. Esta idea de fuerte homogeneidad está en la base de la noción de ciudadano proveniente de la Revolución Francesa.

Al mismo tiempo este período histórico, con su ideología modernista, alentó el desarrollo de las teorías sobre la lengua como un objeto homogéneo, como el estructuralismo y el generativismo. Estas teorías resultaron ser fundamentales para la comprensión de los aspectos cognitivos y estructurales de la lengua, aunque, con algunos énfasis, a veces han sido usadas para oponerse a integraciones políticas (por ejemplo, la creación de dos lenguas donde había una, al instaurarse una frontera política, creando con facilidad dos sistemas lingüísticos donde antes había uno) o pedagógicas (por ejemplo, el descarte de la transferencia de habilidades lectoras u otras entre lenguas en la enseñanza de la lectura por la visión de cualquier lengua extranjera como algo altamente ajeno).

La ideología pluriglósica, en cambio, sostiene otro proyecto, que se podría relacionar con el actual proyecto posmoderno europeo, global y comunitario en el que se defiende y celebra la heterogeneidad, la idea de que la patria está construida en base a las diferencias. El ciudadano europeo de hoy en día es en realidad ciudadan@, multirracial y plurilingüe; lo que lleva como consecuencia a la protección de este plurilingüismo. En los últimos 
tiempos en América también se observa esta preocupación por el respeto de la heterogeneidad lingüística como parte de los llamados derechos civiles (como por ejemplo el black English vernacular o inglés negro) o también derechos de las minorías lingüísticas, con una progresiva oficialización de las lenguas americanas originarias (como por ejemplo el guaraní). Debemos agregar aquí el creciente reconocimiento de las lenguas de señas. Por ejemplo en Uruguay la LSU se ha convertido en una de las lenguas nacionales a través de la Ley $n^{\circ} 17.378$ (URUGUAY, 2001).

La Unión Europea ha hecho un trabajo importante sobre contextos multilingües para formalizar la comunidad en todos los aspectos que tienen que ver con la educación. Observaremos a continuación las ideas sobre educación lingüística que desarrollaron y cómo estas influyen en las metodologías de enseñanza tanto de lengua materna como de segundas lenguas y las diversas habilidades implicadas, como la oralidad y la escritura.

En apartado 1.3. del Marco Común Europeo de Referencia para las Lenguas: Aprendizaje, Enseñanza, Evaluación (CONSEJO DE EUROPA/ DIVISIÓN DE POLÍTICAS LINGÜÍSTICAS, 2002; de aquí en más $M C E L$ ) se discute el concepto de multilingüismo y se propone su sustitución por el de plurilingüismo como marco de enseñanza (e implícitamente de uso) de las lenguas en la Unión Europea. Según el documento referido el multilingüismo "es el conocimiento de varias lenguas o la coexistencia de distintas lenguas en una sociedad determinada" (CONSEJO DE EUROPA; DIVISIÓN DE POLÍTICAS LINGÜÍSTICAS, 2002, p. 4).

Se sobreentiende que esta perspectiva concibe que el conocimiento y uso de cada lengua no se mezcla, o no se debe mezclar, con el conocimiento y uso de las otras lenguas y que en todos los casos debe tenderse al hablante nativo como modelo. Esto se constituye por un lado en una postura política, pero al mismo tiempo está anclado en una perspectiva lingüística y cognitiva de la lengua como una entidad o estructura fuertemente delimitada, opuesta a modelos como el de Hudson (1987).

Es decir, la enseñanza de una segunda lengua o una lengua extranjera, desde esta perspectiva, tiene como objetivo alcanzar la competencia de hablante nativo de dicha lengua, en tanto modelo idealizado que, en definitiva, representa el conocimiento que un hablante oyente ideal tiene de la variedad estándar de la lengua particular que se está enseñando.

Sin embargo, el plurilingüismo supone que: 
conforme se expande la experiencia lingüística de un individuo en los entornos culturales de una lengua, desde el lenguaje familiar hasta el de la sociedad en general, y después hasta las lenguas de otros pueblos (ya sean aprendidas en la escuela o en la universidad, o por experiencia directa), el individuo no guarda estas lenguas y culturas en compartimentos mentales estrictamente separados, sino que desarrolla una competencia comunicativa a la que contribuyen todos los conocimientos y las experiencias lingüísticas y en las que las lenguas se relacionan entre sí e interactúan. En situaciones distintas, una persona puede recurrir con flexibilidad a partes diferentes de esta competencia para conseguir una comunicación eficaz con un interlocutor concreto. Por ejemplo, los interlocutores pueden cambiar de una lengua o un dialecto a otro, explotando así la habilidad que tiene cada uno para expresarse en una lengua y para comprender otra. Una persona puede recurrir al conocimiento de varias lenguas para dar sentido a un texto escrito, e incluso hablado, en una lengua previamente 'desconocida', reconociendo palabras de un fondo común internacional que aparecen con una forma nueva. (...) Desde esta perspectiva, la finalidad de la educación en una lengua queda profusamente modificada. Ya no se contempla como el simple logro del ‘dominio' de una o dos - o incluso tres lenguas- cada una considerada de forma aislada, con el 'hablante nativo ideal' como modelo fundamental. Por el contrario, el objetivo es el desarrollo de un repertorio lingüístico en el que tengan lugar todas las capacidades lingüísticas" (CONSEJO DE EUROPA/DIVISIÓN DE POLÍTICAS LINGÜÍSTICAS, 2002, p. 4 - 5).

Con esta noción de plurilingüismo aparece un radical cambio de enfoque, que plantea numerosas interrogantes al campo de la psicolingüística y la lingüística aplicada, tradicionalmente ligado a una concepción de lengua homogénea y a la existencia de un hablante nativo en tanto conocedor de dicha lengua.

Cabe señalar sin embargo que esta concepción de plurilingüismo hace excesivo énfasis en lo comunicativo: "el individuo no guarda estas lenguas y culturas en compartimentos mentales estrictamente separados, sino que desarrolla una competencia comunicativa". Yendo un poco más allá, podemos considerar en este plurilingüismo las lenguas en su aspecto cognitivo y 
entonces agregar que tampoco las estrategias y tecnologías desarrolladas en una lengua quedan guardadas en compartimientos separados, lo que incluiría estrategias interpretativas, habilidades lectoras etc., y todos estos intercambios entre lenguas, variedades y habilidades darían unidad a la educación lingüística. De esta manera una clase de lectura en una lengua extranjera estaría aportando a las habilidades interpretativas en la lengua o variedad propia, como también lo haría el análisis de una narrativa estructurada a través de otros medios.

El plurilingüismo promueve el uso de muchas variedades lingüísticas, con un equilibrio variable de acuerdo a necesidades, lugares y dominio del hablante de esas variedades y las tecnologías (escritura) que las involucra. A su vez reivindica fuertemente la variedad propia. Esto debería ser no sólo una defensa política sino también cognitiva. Un hablante de español puede hablar en su lengua con un brasileño que habla en la suya, y con ciertas estrategias de convergencia, llegar a entenderse. Lo mismo pasa con la escritura: posiblemente pueda leer en más de una lengua, pero escribir es más difícil. El desarrollo de las habilidades de recepción, comprensión (oral y escrita) son las primeras. En producción oral o escrita, el modelo de procurar precisión "native-like" o similar a nativo, se flexibiliza y se acepta la diferencia de lo no nativo.

La revalorización de la variedad propia conlleva naturalmente a la vez una idealización y una resistencia ante la estándar. La idealización de ese objeto ideal que nunca se alcanza totalmente. La resistencia ante la autoridad de la misma y que, por ende, tiende a coartar la libertad personal ante los usos idiosincráticos.

\section{Reflexiones finales}

En este trabajo hemos presentado algunos asuntos concernientes a la educación lingüística dentro del sistema público de educación en Uruguay, más específicamente concernientes a la lectura y su rol no solo en la educación como sistema sino también en el desarrollo individual, en la producción de ideas propias y en la subjetivación.

Como hemos visto, la lectura es esencial para permanecer y progresar en el sistema educativo. Pero también juega un rol esencial para el individuo en su interacción con el conocimiento y la información ya que es un proceso 
interior, silencioso, de interpretación que encontrará una forma de hacer de ese conocimiento e información algo propio. A veces el individuo quedará más profundamente afectado por el texto. Otras veces perderá o resistirá más la oferta del texto.

Principalmente, nos hemos enfocado en dos poblaciones que han tenido problemas en permanecer y progresar en el sistema educativo: los estudiantes sordos y algunos estudiantes ingresantes a la Universidad. Hemos visto que en general es positivo tratar de intervenir desde el área lengua, más específicamente en la lectura, y reflexionar sobre formas de mejorar las intervenciones del sistema educativo en los procesos lingüísticos. La idea que se ha manejado en toda la investigación aquí presentada es que las prácticas de lectura son clave para mantenerse dentro del sistema educativo, y no así necesariamente ocurre con las prácticas de escribir.

Hemos visto como algunas posiciones teóricas e ideológicas impactan en las intervenciones propuestas por el sistema educativo. Algunas de estas posiciones estimulan actitudes puristas sobre la lengua y dificultan la realización de intervenciones menos tradicionales, mientras que otras cuestionan la lengua como objeto de educación y de uso. Estas posturas conciben el fenómeno del lenguaje en su heterogeneidad, como un conjunto de habilidades o subfenómenos involucrados que tienen que ver con aspectos estructurales o sintácticos, así como con aspectos fonéticos, motores, pragmáticos y otros. Entonces, es posible abrirse a la integración de variedades, por un lado, y a deconstruir las estrategias y habilidades sobre las que se necesite trabajar, como por ejemplo el proceso de la lectura.

Por último, como ya mencionamos, algunas de las asignaturas que enseñan "lengua" también refuerzan ideologías puristas, presentándola como un objeto, homogéneo y bien delimitado. De esta manera estas asignaturas dificultan la enseñanza de la operativa de la lengua, de los sistemas de razonamiento y de metodologías de análisis e interpretación del fenómeno del lenguaje. Reflexionar sobre las ideologías que manejamos sobre la lengua puede abrirnos a nuevas posibilidades a la hora de plantear intervenciones educativas sobre la misma. 


\section{Referencias}

ADMINISTRACIÓN NACIONAL DE EDUCACIÓN PÚBLICA. Documentos de la Comisión de Políticas Lingüísticas en la Educación Pública. Montevideo: Documento Oficial, 2008.

ADMINISTRACIÓN NACIONAL DE EDUCACIÓN PÚBLICA. Primer Foro Nacional de Lenguas de la ANEP. Montevideo: Documento Oficial, 2009.

ADMINISTRACIÓN NACIONAL DE EDUCACIÓN PÚBLICA. Segundo Foro Nacional de Lenguas de la ANEP. Montevideo: Documento Oficial, 2010.

ADMINISTRACIÓN NACIONAL DE EDUCACIÓN PÚBLICA. Tercer Foro Nacional de Lenguas de la ANEP. Montevideo: Documento Oficial, 2011.

ADMINISTRACIÓN NACIONAL DE EDUCACIÓN PÚBLICA. Cuarto Foro Nacional de Lenguas de la ANEP. Montevideo: Documento Oficial, 2012. ADMINISTRACIÓN NACIONAL DE EDUCACIÓN PÚBLICA. Quinto Foro Nacional de Lenguas de la ANEP. Montevideo: Documento Oficial, 2013.

ADMINISTRACIÓN NACIONAL DE EDUCACIÓN PÚBLICA/CONSEJO DE EDUCACIÓN PRIMARIA. Programa de Educación Inicial y Primaria. Montevideo, 2008. Obtenido de: $<$ http://www.ceip.edu.uy/archivos/programaescolar/ProgramaEscolar_14-6.pdf>. Acceso en:24 oct. 2014. ADMINISTRACIÓN NACIONAL DE EDUCACIÓN PÚBLICA/CONSEJO DE EDUCACIÓN SECUNDARIA. Ciclo Básico. Plan 1996. Montevideo, 1996. Obtenido de: $<$ http://www.ces.edu.uy/ces/index.php?option=com_content\&view=article\&id=1085>. Acceso en: 24 oct. 2014.

ADMINISTRACIÓN NACIONAL DE EDUCACIÓN PÚBLICA/CONSEJO DE EDUCACIÓN SECUNDARIA. Plan 2013. Montevideo, 2013. Obtenido de: $<$ http://www.ces.edu.uy/ces/index.php?option=com_content\&view=article\&id=1052>. Acceso en: 24 oct. de 2014.

ARNOUX, E. La lectura y la escritura en la Universidad. Buenos Aires: Eudeva, 2004.

BARCO, S.; LIZARRITURRI, S. Producción y comprensión textual en la universidad. Montevideo: Comisión Sectorial de Enseñanza/UdelaR, 2005. 
BEHARES, L. E. Diglosia escolar: aspectos descriptivos y sociopedagógicos. Trabalhos em Lingüistica Aplicada, Campinas, v. 14, p. 147 - 154. 1989.

; BROVETTO, C.; PELUSO, L. Language Policies in Uruguay and Uruguayan Sign Language (LSU). SignLanguageStudies, Washington, v. 12, n. 4, p. $519-542.2012$. << http://dx.doi.org/10.1353/sls.2012.0008>>

BRUNER, J. Acción, pensamiento y lenguaje. Madrid: Alianza, 1984.

CALDERÓN, J. Teoría y desarrollo de la investigación en educación comparada. México: Plaza y Valdés, 2000.

CARLINO, P. Escribir, leer y aprender en la universidad. Una introducción a la alfabetización académica. Buenos Aires: Fondo de Cultura Económica de Argentina, 2005.

CARTOLARI, M.; CARLINO, P. Leer y escribir en la formación docente: aportes de las investigaciones anglosajonas. Acción Pedagógica, San Cristóbal Edo, n. 21, p. 60 - 79, jan. /dec. 2012.

CISNEROS, M. Lectura y escritura en la Universidad. Una investigación diagnóstica. Pereira: Universidad Tecnológica de Pereira, 2007.

CHOMSKY, N. Aspectos de la teoría de la sintaxis. Madrid: Aguilar, 1965. . El conocimiento del lenguaje. Madrid: Alianza, 1989.

CONSEJO DE EUROPA/DIVISIÓN DE POLÍTICAS LINGÜÍSTICAS. Marco Común Europeo de Referencia para las Lenguas: aprendizaje, enseñanza, evaluación. Madrid: Instituto Cervantes, 2002.

COMISIÓN SECTORIAL DE ENSEÑANZA/UNIVERSIDAD DE LA REPÚBLICA. Relevamiento sobre Desvinculación Estudiantil. Montevideo, 2014. Obtenido de: <http://www.cse.edu.uy/desvinculacion-estudiantil $>$. Acceso en: 24 oct. 2014.

DAWSON, M.; MEDLER, D. Dictionary of Cognitive Science. 2009. Obtenido de: $<$ http://www.bcp.psych.ualberta.ca/ mike/Pearl_Street/Dictionary/ contents/T/topdown.html>. Acceso en: 24 oct. 2014.

DEL VALLE, J. Monoglossic policies for a heteroglossic culture: misinterpreted multilingualism in Modern Galicia. Language and Communication, New York, v. 20, n. 1, p. 105 - 32. 1999. 
DESINANO, N. B. Los alumnos universitarios y la escritura académica: análisis de un problema. Rosario: Homo Sapiens Ediciones, 2009.

DICONCA, B. Desvinculación estudiantil al inicio de una carrera universitaria. Montevideo: CSE/UdelaR, 2011.

HUDSON, R. A. Sociolinguistics. New York: Cambridge University Press, 1987.

LABOV, W. The Social Stratification of Language in New York City. Washington D. C.: Center for Applied Linguistics, 1966.

LARRINAGA, J. A. Deconstructing classroom discourse from a Reading Course. In: TESOL CONVENTION, 50., 2003, Montevideo. Actas del Fifth Southern Cone, Montevideo: URUTESOL, 2003. p. 8 - 10.

; PELUSO, L. Alumnos sordos en la enseñanza media: los profesores de asignaturas humanísticas y científicas como profesores de lengua. In: FORUM INTERNACIONAL DE ENSINO DE LÍNGUAS ESTRANGEIRAS, 3., 2004, Pelotas. [Anais eletrônicos...] Pelotas: UFP, 2004. 1 CD-ROM.

; . Educación bilingüe de los sordos: consideraciones acerca de la escritura, procesamiento del conocimiento y rol docente. Memorias de las XIV Jornadas de Investigación y Tercer Encuentro de Investigadores de Psicología del Mercosur, Tomo III. Buenos Aires: Ediciones de la Fac. de Psicología de la UBA, 2007. p. 464 - 466. ; . Sordera, escritura y enseñanza de lengua. .Memorias del I Congreso Internacional de investigación y práctica profesional en Psicología, XVI Jornadas de Investigación, Quinto Encuentro de Investigadores en Psicología del MERCOSUR. Buenos Aires: Ediciones de la Fac. de Psicología de la UBA, 2009. p. 550 - 552.

LE PAGE, R. V. Problems of description in multilingual communities. Transactions of the Philological Society, Oxford, v. 67, n. 1, p. 189 -212. 1968.

MACRI, E.; NOBOA, L. Análisis descriptivo de la cohorte 2003 que cursó Ciclo Básico en la Facultad de Medicina. Montevideo: UdelaR, 2004.

PARODI, G. Saber Leer. Madrid: Santillana ediciones, 2010. 
PELUSO, L. Sordos y oyentes en un liceo común: investigación e intervención en un contexto intercultural. Montevideo: UdelaR; Psicolibros, 2010.

. Entre la lengua oral escrita y la oralidad de la lengua de señas: buscando los eslabones perdidos. Memorias del III Congreso Internacional de investigación y práctica profesional en Psicología. Buenos Aires: Ediciones de la Fac. de Psicología de la UBA, 2011, p. 342 - 344.

PROGRESA/UNIVERSIDAD DE LA REPÚBLICA. Fundamentos conceptuales de las tutorías entre pares. La experiencia de la Universidad de la República. Montevideo: CSE;UdelaR, 2012.

SAUSSURE, F. de. Curso de Lingüistica General. Madrid: Alianza, 1916,1993.

SCHWEIZER, M. La comprensión lectora en el ingreso universitario. Villa María: Eduvim, 2011.

TERIGE, F.; DIKER, G. La formación de maestros y profesores: hoja de ruta. Buenos Aires: Paidós, 1997.

TORRES, C.; FEDORCZUK, A.; VIERA, A. En diálogo desde la Universidad. Dificultades en la actividad de estudio desde la perspectiva de estudiantes y docentes. Montevideo: UdelaR; PsicolibrosUniversitario, 2009.

TRUDGILL, P.; HANNAH, J. International English. A guide to the varieties of standard English. Routledge: New York, 2013.

UNIVERSIDAD DE LA REPÚBLICA. Universidad de la República. Memoria 2012. Montevideo, 2013. Obtenido de: $<\mathrm{http}: / / \mathrm{www}$.universidad.edu. uy/renderPage/index/pageId/710\#heading_2413>.Acceso en: 24 oct. 2014.

URUGUAY. Ley No. 17.378. Ley de reconocimiento de la Lenguas de Señas Uruguaya -LSU. Montevideo: documento oficial, 2001.

URUGUAY. Ley 18.437. Ley General de Educación. Montevideo: documento oficial, 2008/2009.

Data de registro: $13 / 04 / 2015$

Data de aceite: 26/08/2015 